\title{
Empathy as Key Factor for Successful Intercultural HCI Design
}

\author{
Rüdiger Heimgärtner ${ }^{1}$, Lutz-Wolfgang Tiede $^{2}$, and Helmut Windl ${ }^{2}$ \\ ${ }^{1}$ Intercultural User Interface Consulting (IUIC) \\ Lindenstraße 9, 93152 Undorf, Germany \\ ruediger.heimgaertneraiuic.de \\ ${ }^{2}$ Continental Automotive $\mathrm{GmbH}$ \\ Siemensstraße 12, 93055 Regensburg, Germany \\ \{Lutz-Wolfgang. Tiede, Helmut.Windl\}@continental-corporation.com
}

\begin{abstract}
Successful intercultural communication depends on the personal ability to mutually understand the web of belief of the others using empathic capabilities as shown by empirical examples. Only assuming the perspective of a user by the HCI designer to grasp their needs, can lead to good user interfaces of high usability, thereby evoking excellent user experience. Hence, empathy is a key factor for the successful design of intercultural human computer interaction $(\mathrm{HCI})$.
\end{abstract}

Keywords: Cultural differences, culture, communication, understanding, empathy, intercultural communication, intercultural HCI design.

\section{Problems in HCI Design Caused by Cultural Differences}

Much cultural background has to be considered when designing the functionality and the interaction for global devices [1]: intercultural HCI design comprises significantly more than merely the implementation of a catalogue of requirements for the user interface like considering different languages, colours or symbols. Successful intercultural HCI design goes far beyond a regular design process by taking into account different mentalities, thought patterns and problem solving strategies that are anchored in culture, for example linear vs. non-linear differences [1, 2]. For example, usage patterns which do not occur in everyday life in the source country can arise in the target country due to different power structures [3], for example flat vs. hierarchical ones. Moreover, the designer must know exactly what the user needs or wants (e.g. why, in which context, etc.) [4]. This knowledge can be determined most precisely by using inquiry approaches or methods based on communication [5]. Just using observation techniques or relying on expert opinions results in less reliable information although these results are very useful, too. However, problems in intercultural communication, particularly those in requirement analysis, inhibit good usability for system design and the related user experience. Therefore, in order to improve cooperation with designers, managers, users and customers, the problems of comprehension must be analysed. 


\section{Solving Problems in Intercultural HCI Design by Successful Intercultural Communication Based on Empathy}

Several levels of intercultural know-how contribute to successful intercultural HCI design. The communication level constitutes the basic level, followed by the levels of project management, software and usability engineering and HCI design itself on the way to successful intercultural HCI design (cf. Figure 1).

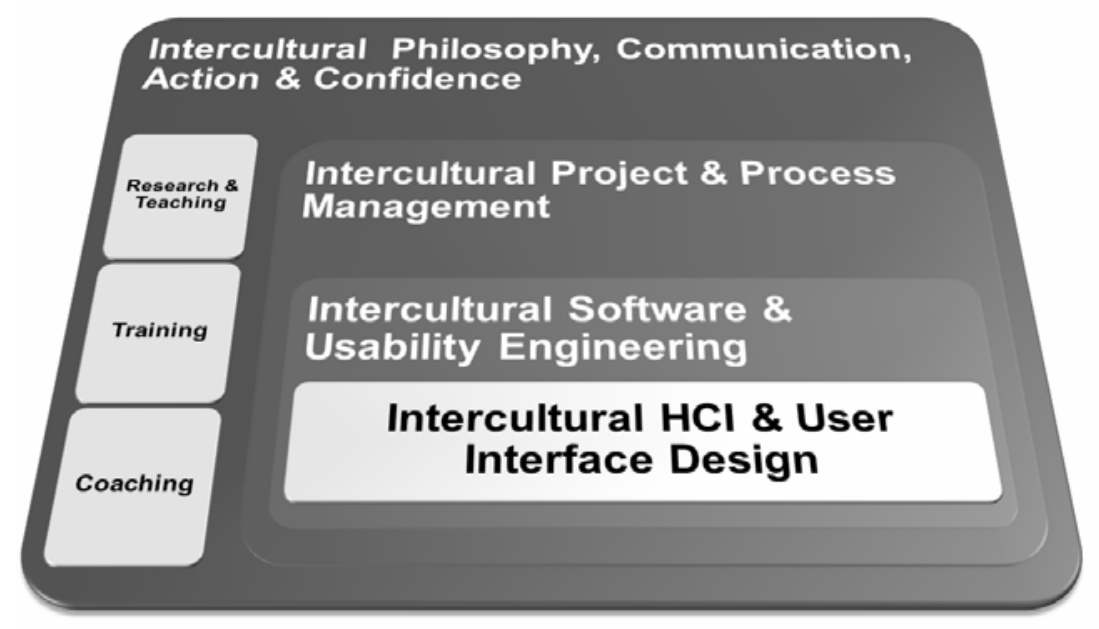

Fig. 1. Levels on the Way to Successful Intercultural HCI Design (Source: IUIC)

Hence, on all levels (strongly influenced by the philosophy of the respective cultures), intercultural communication skills at the basic level can contribute to the solution of problems raised on the upper levels by cultural differences.

\subsection{Intercultural Communication Requires Mutual Understanding}

For successful (intercultural) usability engineering, an adequate engineering process is necessary to ensure good usability (i.e. when the user understands the developer's device and is thus able to easily operate it satisfactorily), it is necessary that the developer understands the user [2, 6], because they have different points of view [7]. At least the following aspects of the user must be analysed in detail before the product can be developed:

- World view, Weltanschauung (metaphysical approach) of the end-user,

- General knowledge (procedural and factual knowledge) of the end-user,

- The context in which the product will be used by the end-user,

- The tasks the end-user intends to accomplish by using the product. 
Only by considering these aspects, intercultural communication as an essential prerequisite for intercultural usability engineering, user interface design, and user experience will it be successful and can it lead to successful international product design.

\subsection{Empathy as a Prerequisite for Mutual Understanding}

Successful communication depends crucially on the capability for empathy of the people involved $[8,9,10,11]$. Communication without empathy does not deliver the desired results $[12,13]$. This in turn assumes a certain level of confidence and trust (e.g. Principle of Charity, cf. [8]) and includes the knowledge of how to read between the lines of the counterpart's communication depending on culturally different rules. This includes the usage of linguistic rules, for example, Austin's felicity conditions or Gricean maxims $[9,21]$. Therefore any literal translation of a conversation is prone to misinterpretation since the extension of the concepts can be different in different cultures ("linguistic relativity", cf. [14]). As context must also be taken into consideration, it is important to consider these aspects in communicating and focus on the intellectual horizon of the communication partner as widely as possible. This can occur in particular through personal and on-site communication and is particularly difficult over the phone due to the absence of mimical and gestical signals. Even more problems arise in intercultural communication compared to intra-cultural communication due to differing world views and the context in which clarification occurs. For this reason, the empathic capability to put oneself in someone else's situation is particularly important. The application of empathy in the end contributes to a successful communication supporting a mutual linguistic code. In particular, intercultural user experience designers must be able to put themselves in the position of the user in order to know and understand his or her intentions and needs, to ideally experience them, and to implement them in the product.

In order to build up not only understanding but also the ability to put oneself in someone else's position, it is initially necessary to be on the same wavelength to find a connection to the other person. This requires the alignment of communication coding (vocabulary and grammar) and to achieve a situation where the other person wants to communicate. Thus a relationship is built up in such a way that future communication remains possible. If this connection is given, it is important to preserve access to the other person's knowledge base ("Web Of Belief", cf. [15]) using a mutual topic of conversation in order to examine the knowledge base of the counterpart in regard to extent, type, and quality. Only then it is possible to find the right "hook" in further conversations and consequently "fetch up" the other person's web of belief at the most relevant point to quickly pick up the same wavelength again. The web of belief contains beliefs and desires derived from premises, assumptions and facts using logical rules and are recursively formed by experience from birth. Through training intercultural competency, approaching the web of belief of a member of other cultures is possible. Thereby, exchange of experiences is very effective, trust can be conferred from one person to another by introducing the persons and critical interaction situations [16] can be weakened.

This works, if it is clear how the other person thinks (i.e. what world view he or she holds i.e. which premises and assumptions about the world he or she has). This is necessary in order to make choices which are relevant to the job at hand and correct 
for successful communication with a continually expanding set of extra information. This is particularly the case in intercultural contexts. The ability to assess and understand the person's thinking patterns enables an adequate reaction to the people involved. In the same way, the leading and guiding of conversation, e.g., as facilitator or investigator is successfully supported.

\subsection{Types of Empathy and Scale of Empathic Sensibility}

There are several types of empathy (cf. e.g. [12]):

- Hypothetical empathy by scaling oneself into fantasies to act virtually (e.g. thought experiments),

- Cognitive empathy by changing the thinking perspective by assuming the perspective of another person to see their world with one's eyes,

- Emotional empathy by taking on the concerns of others to sense their feelings,

- Personal distress as the capability of being affected by other human beings in problematic emotional situations or danger,

- Determined empathy as a means to reach a certain purpose (e.g. coping behaviour to become a member of a group),

- Moral empathy applied to manage mutual benefit (e.g. for better understanding or successful communication)

Empathy is related to emotional and social intelligence as well as to tactfulness and altruism.

However, empathy also presupposes the capability to separate oneself from other persons to get the chance to recognize the differences to them and then to put oneself in their position. Within the intercultural context, this requires being aware of one's own cultural standards before it is possible to compare and recognize differences to other cultures [16].

\subsection{How to Achieve Empathy?}

How can problems in intercultural communication be resolved through empathy? Problems in intercultural communication manifest themselves in critical interaction situations, in which the expectations of at least one party in the interaction are disappointed and can thus result in unease, friction or indeed conflict [16]. Critical interaction situations (CIS) are resolved through cognitive empathy, in other words the ability to see a situation from the other's perspective; to enter the other's cognitive world, by understanding and assuming the other's web of belief for this purpose. How is this possible? The following steps play an important role:

0 . If the other person is entirely unknown, initial interpretation is radical: his or her web of belief must be construed almost entirely from scratch [cf. 19].

1. Analyzing the other's output such as body language, actions and statements begins to reveal aspects of the other's web of belief.

1.a. In the absence of external stimuli, this analysis is based largely on observation, for example of behavior (actions), appearance, the effects of actions or behavior and 
verbal, nonverbal, and written communication (statements, facial expressions, gestures, documents, etc.).

1.b. In the presence of external stimuli, the analysis may draw on:

1.b.1 questions and an analysis of the output;

1.b.2 an analysis of the output after presenting the counterpart with a specific situation (e.g. a critical interaction situation (CIS)).

The following steps are required after analysis:

2. Generation of a cognitive model (web of belief) of the counterpart

3. Assimilation of the cognitive model of the counterpart

4. Doing step 1 from the counterpart's perspective (in the specific situation)

5. Understanding the counterpart's output (on the basis of the specific situation)

6. Analysis of the specific situation and its root causes

7. Assimilation of the optimal cognitive model by retracing the root causes and retracting the steps which led to the specific situation.

8. Doing step 1 from the counterpart's perspective (in the above specific situation)

9. Comparison of the output with the output in 5 .

10. If the output is desirable compared to 5, i.e. positive (no negative actions, friction, etc.), the optimal cognitive model can then be communicated to the counterpart. Steps 1-10 must otherwise be repeated until the desired (positive) output is achieved.

The optimal cognitive model is communicated to the counterpart in the following stages:

a) Assimilation of the cognitive model of the counterpart.

b) Explanation of each step retracted to achieve an optimal cognitive model of the counterpart.

c) Putting the counterpart in one's own place through a) (in the specific situation).

d) The counterpart understands positive output despite the CIS.

If the counterpart is a member of a foreign culture, "Step 5: understanding the counterpart's output (on the basis of the specific situation)" can only be achieved by being trained in intercultural communication (e.g. by workshops, foreign experience, living abroad etc.).

\section{Examples for the Necessity of Empathic Capabilities}

The following examples refer particularly to experience with projects in the automotive supplier industry concerning China and Germany.

\subsection{Greater Project Success by "Guanxi”}

The communication between Chinese and Germans is initially so difficult because the rules about how to interpret and understand the utterance of the counterpart, located in the web of belief of the other, are not known. For example, calling a Chinese for support is problematic because the structure, hierarchy and the related rules in China are not known to Germans. The Chinese must strictly follow the rules of the hierarchy in order to support the German's demands and suffers the dilemma of giving support 
without losing face within his own organization or impeding the good relationship to the one requesting support [18]. In this sense, the Chinese cannot satisfy the request of the German participant without losing face [21]. Therefore, nothing happens. This example will be explained to offer an impression of the difficulty to understand the various effects of the complex relationships in China ("guanxi" [17]). Figure 2 illustrates a part of the structure of a Chinese team hierarchy as well as the relationships between the team members.

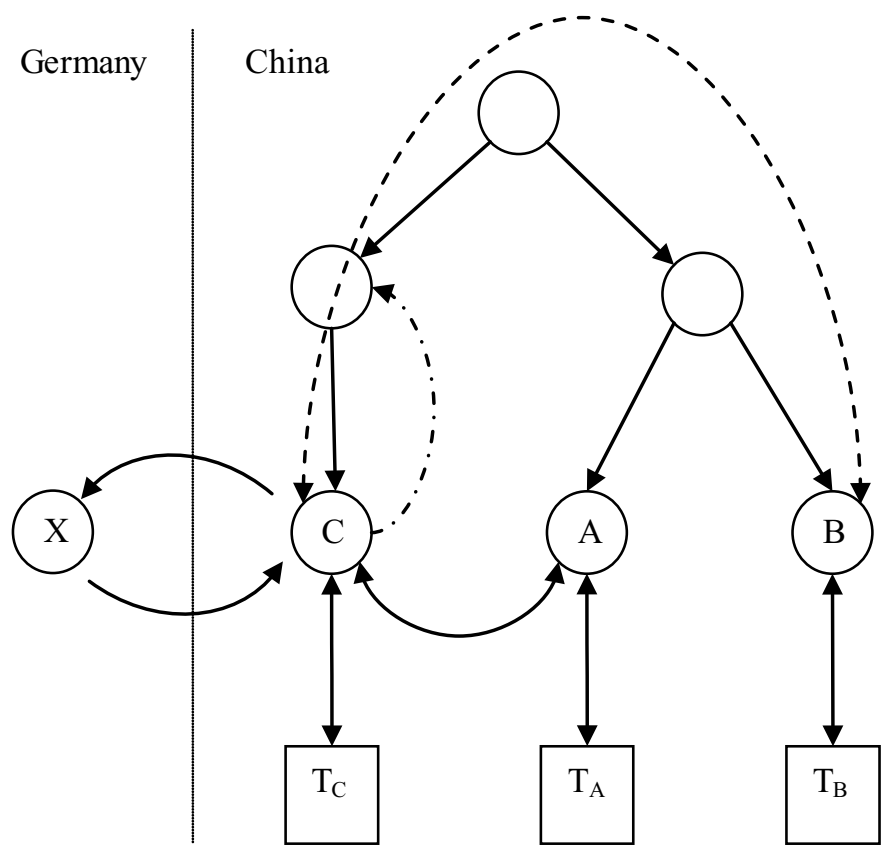

Fig. 2. Chinese Team Hierarchy

Imagine that a German team/project leader $\mathrm{X}$ requests support from the Chinese project team $\mathrm{T}_{\mathrm{C}}$. Now, the team/project leader $\mathrm{C}$ knows: "Our team $\mathrm{T}_{\mathrm{C}}$ need to support $\mathrm{X}$." and communicates to $\mathrm{X}$ : "Yes, our team $\mathrm{T}_{\mathrm{C}}$ will support you.". However, say to ensure fully the requested support for $\mathrm{X}, \mathrm{C}$ needs also to ask the team leaders $\mathrm{A}$ and $\mathrm{B}$ from the teams $T_{A}$ and $T_{B}$ providing sub-project support for team $T_{C}$. Say $C$ provides support to $X$ as best as he and his team $T_{C}$ as well as the support from team $T_{A}$ can provide. However, $C$ does not provide support from team $T_{B}$ to $X$. $X$ is not satisfied with the situation and does not understand why he is not supported fully as requested. What is the reason for this undesired situation?

On the one hand, C and A have a good (personal) relationship ("guanxi"), e.g. because they had been at the same university. Therefore, $\mathrm{C}$ can ask A for support and bypasses the strict hierarchy rules. On the other hand, $\mathrm{C}$ is not connected very well via "guanxi" to B. Therefore, he must go via the official path to B following the whole hierarchy (indicated by the dotted arrow in Figure 2). The hierarchy process is defined 
by transfer orders "top-down". By this, C's responsibility does not include sending requests to his boss or to his boss's boss because it is not part of his role (represented by the chain-dotted arrow in Figure 2). Hence, he cannot do the request according to his job definition and the given responsibility to him. Furthermore, he does not want to "lose face" in front of X by explaining to him his inability to fulfill the task. As a result, the information passed to $\mathrm{X}$ is incomplete. Although, $\mathrm{X}$ is aware of the requested support/dependencies of the teams $T_{A}$ and $T_{B}$, he cannot start any action like requesting top management support due to missing feedback about why $\mathrm{T}_{\mathrm{A}}$ can support and $\mathrm{T}_{\mathrm{B}}$ cannot. In principle, the same situation arises in Germany and it is also a known communication issue between German teams - but the details are different. In any case, empathic capabilities are particularly required to put oneself into the situation to be able to master it.

\subsection{Better Understanding}

Quite often the Germans complain that the Chinese speak "poor English". To understand Chinese people and vice versa it requires a great deal of context. However, German people normally do not know the Chinese context and therefore they do not even understand the questions of the Chinese counterpart. Hence, on the one hand, answers given by Chinese people are often assumed to be incorrect because the context between the explicit statements is not understood by Germans. For example, a Chinese person will always answer in order not to lose face. Thus, a non-Chinese person should not be surprised by answers, which cannot be understood because it seems they do not fit to the question. In this case, one should put oneself in the Chinese's position and consider why the answer is so unusual as well as analyse, which content needs to be transferred to enable the colleagues in China to understand the question. On the other hand, our direct and open answers probably shock Chinese people, because in their eyes, i.e. from the Chinese perspective, we lose face. However, for Germans, losing face is not as important as for Chinese, which seems to Chinese that Germans lose face without batting an eye. This puzzles Chinese people and alienates them. Empathy is needed.

\subsection{Principle of Sustainability}

German engineers "like" to improve things. By giving such a request, a Chinese college will not understand why he should develop products of higher quality for "tomorrow" when the customer accepts the quality of the things being sold "today". This phenomenon roots in the Chinese culture: the Chinese are people of today who are typically pragmatic and functionally attuned without strongly focussing on sustainability. Therefore, a good deal of empathy is also desirable here.

\subsection{Time-Saving and Problem-Solving by Knowing Relation Networks}

Sometimes, projects proceed poorly because one neither knows or shares each other's problems nor is able to solve them. This is because the Chinese network of relations is too difficult to understand in order to always find the right behavioural solutions. Outsiders can, however, certainly get an understandable view of the situation when they have access to very high linguistic and social expertise. In order to be able to understand this network from the inner perspective, however, one needs not only to be 
born into this perspective, i.e. to be a Chinese, but additionally one needs the understanding by the Chinese that there are complex individual networks and how they can and must be used by the employees. Therefore, in order to understand and consider the importance of this network of relations, people from the target market should be represented in the project team. However, probably even a Chinese cannot get along with the entire Chinese network from an outer perspective.

\subsection{Removal of Prejudice for Successful Explanations}

The risk of prejudice is very strong when one is in a foreign culture: the impressions are very intensive but also very easily afflicted with prejudices, which, without corresponding reflection, quickly become generalisations. If one has been in a foreign culture for a very long time, i.e. one puts oneself in the position of the other people for a long time, then one's impressions are weaker but one's judgements are more ingrained and finer while taking into account more of the contextual background. Through regular reflection, stereotypes are reduced and attempts to provide explanations for critical interaction situations are more successful. A similar, but much weaker effect can be reached, if there is a chance to establish a multicultural team. The team members can learn from each other if they are facilitated with empathic capabilities (including willingness).

\section{Applying Empathic Capabilities in Intercultural HCI Design}

Global User Interfaces, which would suit all culture domains, users and contexts, do not yet exist, at least for technical if not for more fundamental reasons. Computers do not yet possess empathy (cf. the so-called ,hard problems of AI“ [22, 23]. At the moment computers lack environmental data (through sensors), the complex processing patterns and the respective knowledge of the world needed to develop empathy. Furthermore, the cultural differences involved in the interaction of the user with the system must be integrated in such knowledge of the world so that the system can adjust for it respectively. Finally, even if these challenges were met the so-called 'bootstrapping' problem of adaptive systems would remain. Because the system is not yet acquainted with the user on his first encounter, the system cannot adapt to him. It is a matter of time until the system gets acquainted with the user and can adapt itself to him. At least the following areas must be considered in HCI, i.e. HumanComputer-Interaction: task, context/situation and tools used [24]. In this case the cognitive processes of the user differ from the results of studies or discrete situations due to his cultural and environmental conditioning and personal experience. The concept of the task intended (as well as the task itself) is no longer congruent. That requires the system (computer / machine / tool) to adapt as perfectly as possible to many different aspects, which however has not yet been possible to implement because of the multitude of aspects and the resultant complexity.

As long as the above mentioned problems have not been completely solved, human beings must accordingly attune HCI to the intended cultural domain, user group and context [25]. To do so, the HCI designer must be able to immerse himself in these cultural domains, user groups and contexts in order to extract the relevant requirements for the HCI design. 
Hierarchical thought patterns (cf. section 3.1) can exercise an effect on menu structures (i.e. flat vs. deep or highly linked vs. loose) or access rights. Better understanding of the context (cf. section 3.2) leads to better situational adaptation in HCI (i.e. avoiding loss of face through negative feedback in system messages). Understanding networking relations (cf. section 3.4) better facilitates the use of virtual agents (anthropomorphic characters) in Asiatic information systems. Impartiality (cf. section 3.5 ) in the approach to , usability engineering' augments its success and reduces the complexity of the HCI because no detours must be anticipated. By avoiding prejudices unnecessary effort can be circumnavigated (e.g. avoiding the color yellow in China due to association with "red-light-district" connotations). If a situation is known, no prejudices crop up but rather proper evaluations develop, which speeds up the design process and make it specific. That is the way concrete, goal oriented and adaptive HCI design develops and thereby yields highly serviceable and user attuned HCI.

\section{Challenges by Empathy}

The effort required to train and make use of one's empathic capabilities is a task that is to be taken seriously. How much effort is required over and beyond the use of one's empathetic capabilities? None! As empathic capabilities, however, are not equally distributed nor easily trained in all people, it seems likely that a career as a user experience designer with the goal of achieving successful (intercultural) HCI design is not suited to everyone.

The question of whether the problems in understanding and communicating are more individual empathic problems or based on cultural differences remains open. The question "who gets on well with whom" must still be explicitly explored in relevant studies. It is, however, clear that for communication to be successful, both communication partners in a conversation must be open to each other and in this way be able to use certain basic empathic skills.

\section{Conclusion}

Empathy is an essential prerequisite for successful intercultural communication, which promotes successful intercultural HCI design and intercultural usability engineering and, as a consequence, good user experience. Hence, it should be ensured and promoted that usability engineers receive, know and apply empathy. This paper just touches the problems, which should be researched in detail in further studies.

\section{References}

1. Röse, K.: Methodik zur Gestaltung interkultureller Mensch-Maschine-Systeme in der Produktionstechnik. Univ., Kaiserslautern (2002)

2. Honold, P.: Interkulturelles usability engineering: Eine Untersuchung $\mathrm{zu}$ kulturellen Einflüssen auf die Gestaltung und Nutzung technischer Produkte. VDI Verlag, Düsseldorf (2000) 
3. Hofstede, G., Hofstede, J.G.: Cultures and Organizations: Software of the Mind, 2nd edn. McGraw-Hill, New York (2005)

4. Holzinger, A.: Usability engineering methods for software developers. Commun. ACM 48(1), 71-74 (2005)

5. Rubin, J., Chisnell, D.: Handbook of Usability Testing. How to Plan, Design, and Conduct Effective Tests. and Conduct Effective Tests. Wiley Publishing Inc., Indianapolis (2008)

6. Nielsen, J.: Usability Engineering. Kaufmann, Amsterdam (2006)

7. Eigenbrode, S., Wulfhorst, J.D., Althoff, D.M., Golberg, C.S., Merrill, K., Morse, W., Nielsen-Pincus, M., Stephens, J., Winowiecki, L., Bosque-Perez, N.A.: Employing Philosophical Dialogue in Collaborative Science. BioScience 57, 55-64 (2007)

8. Dennett, D.: The Intentional Stance. MIT Press, Cambridge (1987)

9. Austin, J.L., von Savigny, E.: Zur Theorie der Sprechakte. Reclam, Stuttgart (2002)

10. Searle, J.R., Kiefer, F., Bierwisch, M.: Speech act theory and pragmatics, pp. 291-312. Reidel, Dordrecht (1980)

11. Schwartz, S.H.: Universals in the content and structure of values: theoretical advances and empirical tests in 20 countries. Advances in Experimental Social Psychology 25, 1-65 (1992)

12. Stueber, K.: Rediscovering Empathy: Agency, Folk Psychology, and the Human Sciences. MIT Press, Cambridge (2006)

13. Stueber, K.: Empathy. Stanford Encyclopedia of Philosophy (2008), http://plato. stanford.edu/entries/empathy

14. Whorf, B.L.: Sprache, Denken, Wirklichkeit. Beiträge zur Metalinguistik und Sprachphilosophie 25. Aufl., Reinbek bei Hamburg (2008)

15. Quine, W.V.: Word and Object. MIT Press, Cambridge (1960)

16. Thomas, A.: Psychologie interkulturellen Handelns. Hogrefe, Göttingen (1996)

17. Budery, R., Huang, G.T.: Guanxi (The art of relationships). Simon \& Schuster, New York (2006)

18. Brislin, R.W., Yoshida, T.: Intercultural communication training: An introduction. SAGE Publications, Thousand Oaks (1994)

19. Davidson, D.: Belief and the Basis of Meaning. In: Davidson, D. (ed.) Inquiries into Truth and Interpretation, pp. 141-154. Oxford University Press, Oxford (1984)

20. Grice, P.: Studies in the way of words. Harvard Univ. Press, Cambridge (1993)

21. Victor, D.A.: International business communication. Harper Collings, NY (1998)

22. Görz, G.: Handbuch der Künstlichen Intelligenz. Oldenbourg, München (2003)

23. Winograd, T., Flores, F., et al.: Erkenntnis, Maschinen, Verstehen: Zur Neugestaltung von Computersystemen. Rotbuch Verl., Berlin (1989)

24. Herczeg, M.: Software-Ergonomie: Grundlagen der Mensch-Computer-Kommunikation. Oldenbourg, München (2005)

25. Van Kleek, M., Shrobe, H.E.: A practical activity capture framework for personal, lifetime user modeling. In: Conati, C., McCoy, K., Paliouras, G. (eds.) UM 2007. LNCS (LNAI), vol. 4511, pp. 298-302. Springer, Heidelberg (2007) 